\title{
College Members whose deaths were reported at Council meetings between October 2015 and October 2016
}

(C) 2017 The College. This is an open-access article published by the Royal College of Psychiatrists and distributed under the terms of the Creative Commons Attribution License (http://creativecommons.org/licenses/by/4.0), which permits unrestricted use, distribution, and reproduction in any medium, provided the original work is properly cited.

Adabie, Kenneth Horn, Affiliate, Croydon, London, UK Al-Hindawi, Amir Abdul Amir Ali, Affiliate, Rotherham, South Yorkshire, UK

Arshad, Muhammad, Affiliate, Cheadle, Cheshire, UK

Baker, Ronald Stewart, Fellow, Southwold, UK

Barker, Montagu Gordon, Fellow, Bristol, UK

Barker, Philip, Fellow, Calgary, Alberta, Canada

Betts, Timothy, Fellow, Birmingham, UK

Bhate, Suryakant Ramji, Fellow, Newcastle upon Tyne, UK

Browne, Elizabeth Foster, Foundation Member, London, UK

Burrows, Graham Dene, Fellow, Richmond, Victoria, Australia

Burvill, Peter Walter, Fellow, Como, Western Australia,

Australia

Carney, Michael William Patrick, Fellow, Bathampton, Bath, UK

Cheyne, Alexander lan, Fellow, Kippen, Stirling, UK

Choudhary, Prasoon Chandra, Fellow, Aberdare, UK

Davies, Stephen Lewis, Member, Breaston, Derby, UK

Denson, Raymond, Member, Thunder Bay, Ontario, Canada

Discombe, Anne-Marie, Member, Glasgow, UK

Edmonstone, Yvonnne Grace, Fellow, Inverness, UK

El Gorashi, Gamal El Din Suleiman, Member, Canterbury, UK

Fielder, Michael Hugh, Member, London, UK

Forrest, Derek William, Fellow, Wirral, UK

Frame, Archibald Hamilton, Fellow, Glasgow, UK

Galea, Abraham, Fellow, Mosta, Malta

Galley, Margaret Walton, Foundation Affiliate, Castletown, Isle of Man

Galley, Wilfred, Foundation Affiliate, Castletown, Isle of Man Gay, Martyn John, Fellow, Newton Poppleford, Sidmouth, UK Gosall, Gurpal Singh, Member, Brockhall Village, Old Langho, Blackburn, UK

Graham, Alexander John, Fellow, Law, Carluke, UK

Gundy, Greville Henry, Member, South Croydon, London, UK Hall, Peter, Fellow, Upton upon Severn, UK

Halpenny, John Vincent, Member, Swords, Co Dublin, Ireland Hanmanthraya, Bheemraya, Member, Durham, UK Hassan, Imad Mohamed Babiker, Affiliate, London, UK Hervey, Morag Stuart, Fellow, Wells, UK

Hinton, John Mark, Fellow, Leigh, Sherborne, UK

Hocking, Frederick H., Fellow, Melbourne, Victoria, Australia Hordern, Anthony, Fellow, North Turramurra, New South

Wales, Australia

Hughes-Roberts, Huw Elfyn, Fellow, Prestatyn, UK

Jones, Eve, Member, Mellor, Blackburn, UK

Kay, David William Kilbourne, Fellow, Newcastle upon Tyne, UK

Kerry, Raphael James, Fellow, Sheffield, UK

Lipscomb, Colin Francis, Member, Qualicum Beach, British

Columbia, Canada

Mahapatra, Samarendra Nath, Fellow, Billingham, UK
MacLean, Rona, Fellow, Ashtead, UK

Magapu, Viswanathan, Member, Marchington, Staffordshire, UK Marks, John, Fellow, Duxford, Cambridge, UK

Martlew, Robert Howard, Fellow, Wallasey, Merseyside, UK McGauley, Gillian Anne, Fellow, London, UK

McGregor, Charles Malcolm, Foundation Affiliate, Doncaster, UK

McPherson, Angus Francis, Member, Findon, Worthing, UK

McQuaid, Arthur, Fellow, Cambridge, UK

Middlefell, Robert, Fellow, Raby Mere, Wirral, UK

Milner, George, Fellow, Worcester, UK

Nazir, Zoonia, Member, Birmingham, UK

Noble, Rochelle Fredelene, Affiliate, Salford, UK

Nwulu, Bernard Nchewa, Fellow, Rotherham, UK

O’Brien, James (Jim), Member, King's Lynn, Norfolk, UK

O'Malley, Patrick Pearse, Fellow, Booterstown, Co Dublin, Ireland O'Sullivan, Mary Teresa Martin, Member, Baldrine, Isle of Man Pargiter, Russell Ashby, Fellow, Hobart, Tasmania, Australia Prins, Herschel Albert, Mental Health Associate, Houghton on the Hill, Leicester, UK

Rae Grant, Naomi Ingrid, Fellow, North York, Ontario, Canada Rae-Grant, Quentin Alexander Frain, Fellow, Toronto, Ontario, Canada

Read, Priscilla Elise, Fellow, Layer Breton, Colchester, UK

Rosen, Bernard Keith, Fellow, London, UK

Satkunanayagam, Vaithianathan, Fellow, Epsom, UK

Schnieden, Vivienne, Fellow, Paddington, New South Wales,

Australia

Scott, Jeremy Peter Dixon, Member, Lillte Petherick,

Wadebridge, Cornwall, UK

Silk, Kenneth R., International Associate, Ann Arbor, Michigan, USA

Silverman, Maurice, Fellow, Whitefield, Manchester, UK Smith, Alfred Leonard Gordon, Fellow, Lindley, Huddersfield, UK Speed, Dorothy Elizabeth Maud, Foundation Affiliate, Rode, Frome, UK

Thomas, David John, Member, Dudley, West Midlands, UK Thomas, Mira David, Member, Bathgate, West Lothian, UK Thompson, Ruth, Board of Trustees Committee Member, London, UK

Tychopoulos, Gregory, Member, Attikis, Athens, Greece Walton, John Nicholas, Honorary Fellow, Belford, UK

Waters, Thomas Cyril, Member, Newent, Gloucestershire, UK Wells, Joan Catherine, Member

Wilson, Alan Hamilton, Fellow, Bessacarr, Doncaster, UK

Younas, Ayesha, Affiliate, Bolton, UK

Zealley, Andrew King, Fellow, Edinburgh, UK

doi: $10.1192 / p b .41 .1 .60$ 